\author{
Military Technical College \\ Kobry El-Kobbah, \\ Cairo, Egypt.
}

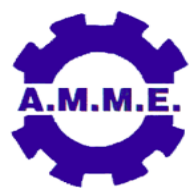

$16^{\text {th }}$ International Conference on Applied Mechanics and Mechanical Engineering.

\title{
IMPROVEMENT OF RESIDUAL STRESSES IN THICK WALL CYLINDERS USING MULTIPLE AUTOFRETTAGE
}

\author{
O. R. Abdelsalam*
}

\begin{abstract}
Autofrettage and Re-autofrettage processes have been used to generate compressive residual stress at the near bore area of the cylinder to enhance the load carrying capacity of the cylindrical pressure vessels. These processes' main drawback appears in the detrimental high tensile residual stress at the outer part of the cylinder. On the basis of these findings, internal surface autofrettage combined with external surface autofrettage processes have been used to improve not only by increasing of compressive residual stress at the near bore area but also by decreasing of tensile residual stress at the outer surface area. For these cyclic plasticity processes, an accurate finite element model governed by non-linear kinematic hardening model (Chaboche model) has been used. This model simulates the measured stress-strain actual behavior for NiCrMoV125 steel alloy.
\end{abstract}

\footnotetext{
* Egyptian Armed Forces.
} 


\section{INTRODUCTION}

Few researchers have attempted to use what they called re-autofrettage. This is a procedure wherein the pressure vessel is autofrettaged once and then, without any intervening process, is subjected to a second similar autofrettage cycle. This process may enhance the compressive residual stresses in the near bore area of the cylinder.

Parker [1] investigated double and triple autofrettage processes for his thick-wall cylinder to mitigate the Bauschinger effect. It has been found that the fatigue life of the cylinder is improved by at least a factor of 2. Jahed et al. [2] used the same technique implemented by Parker [1] but the autofrettage pressure was changed for each cycle. They found that there is no benefit in the re-autofrettage of a tube with the same autofrettage pressure, and the real benefit comes from using a low autofrettaged pressure first then a higher one. Compound autofrettaged and shrinkfitted cylinders have been also used to increase the beneficial compressive residual stresses at the near bore area of the cylinder [3-6]. As discussed, the previous investigations were mainly focused on inducing beneficial residual stresses at the near bore area, neglecting the outer part. However, the results show that autofrettage induces harmful tensile residual stress at the outside layer of the cylinder, which may affect the fatigue life and carrying load capacity of the cylinder. Parker [7] proposed a compound cylinder the inner layer subjected to inner autofrettage load, while the outer layer subjected to reverse autofrettage process by applying the autofrettage load at the outer surface, and then shrink fit the two layers together. Ossama and Sedaghati $[8,9]$ proposed a double autofrettage process, in which a cycle of external surface autofrettage prior to an internal autofrettage cycle takes place. They improved the residual stress for a compound two layer cylinder by increasing the beneficial compressive stresses at near bore area and also decreasing the detrimental tensile stresses at the outer part of the cylinder.

On the other hand, many models have been used to model the autofrettage process considering Bauschinger effect. Jahed and Ghanbari [10] measured the actual tensile-compressive stress-strain behavior of alloy steel during the autofrettage process. They found that there is $30 \%$ over-estimation of compressive residual stress when compared with the ideal and bilinear isotropic hardening model. Huang [11] proposed a general autofrettage model that integrates the material strainhardening relationship and the Bauschinger effect, based upon the actual tensilecompressive stress-strain curve. Huang and Moan [12] proposed an analytical model based on the actual tensile-compressive curve of the material and the vonMises yield criterion incorporating the Bauschinger effect factor and the unloading modulus variation as a function of prior plastic strain. Amin et al. [13] found the optimum autofrettage pressure for a thick wall cylinder using multi-kinematic hardening model. Farrahi et al [14] studied the effect of re-autofrettage process on the residual stresses for thick wall cylinder using non-linear kinematic hardening model. They found that re-autofrettage without heat treatment only improves the residual stresses in high autofrettage percentage, but for low autofrettage percentage this method has no benefit.

In this paper, new techniques are examined by applying an autofrettage cycle on the external surface of the cylinder prior to different autofrettage cycles on the internal 
surface of the cylinder. Also combinations of double autofrettage processes followed each other applied to a mono-block cylinder are investigated. The residual stresses for these new combinations have been evaluated using nonlinear kinematic hardening finite element model and then compared together.

\section{KINEMATIC HARDENING MODELS}

Analysis procedure for autofrettage process involves autofrettage pressure and overstrain (defined as the proportion of the wall thickness of the tube which behaves plastically during the initial application of autofrettage pressure) which is typically based on Tresca or von Mises failure criteria. Here, using two different kinematic hardening models approximating the measured real material behavior (NiCrMoV125 steel) [10]. The first model is the bilinear kinematic hardening model in which $E$ is the slope of the straight line in the elastic region (modulus of elasticity) and $\mathrm{H}$ is the slope of the straight line in the plastic region as shown in Figure 1.

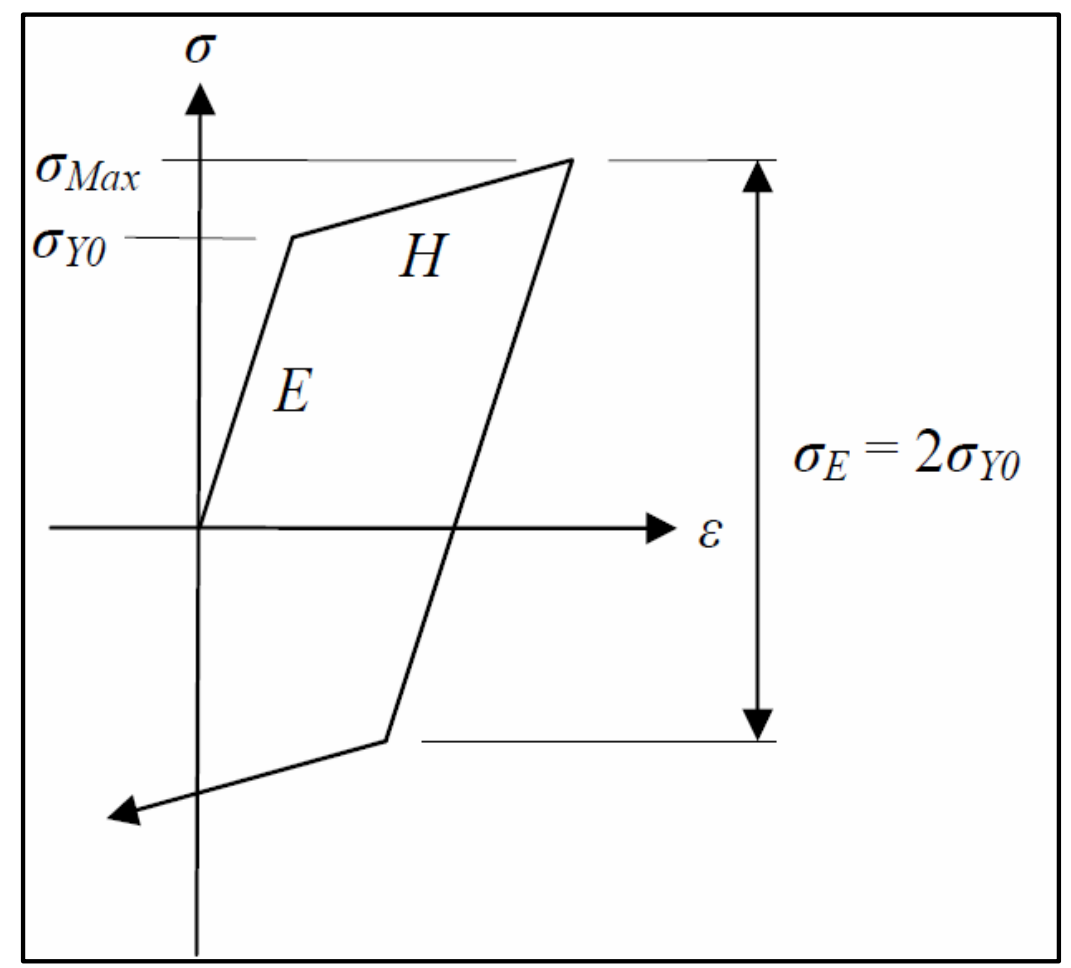

Figure 1: Bilinear kinematic hardening behavior.

On behalf of the second model, a non-linear kinematic hardening model (Chaboche), as shown in Figure2, has been utilized for accurate approximation of the real stressstrain behavior under cyclic load, specially the progressive accumulation of the plastic strain during cyclic loading. The used model has been proposed by Chaboche and his co-workers [15-17]; this model is based on a decomposition of non-linear kinematic hardening rule which is mainly significantly described in three critical segments of a stable hysteresis curve. These three segments are: 1 . the initial modulus when yielding starts, 2. the nonlinear transition of the hysteresis curve after yielding starts until the curve becomes linear again, 3. the linear segment of the 
curve in the range of higher strain. It also enables the description of: a) the nonlinearity of stress-strain loops, under cyclically stable conditions, b) the Bauschinger effect, and c) the cyclic hardening and softening of materials with asymptotic plastic shakedown.

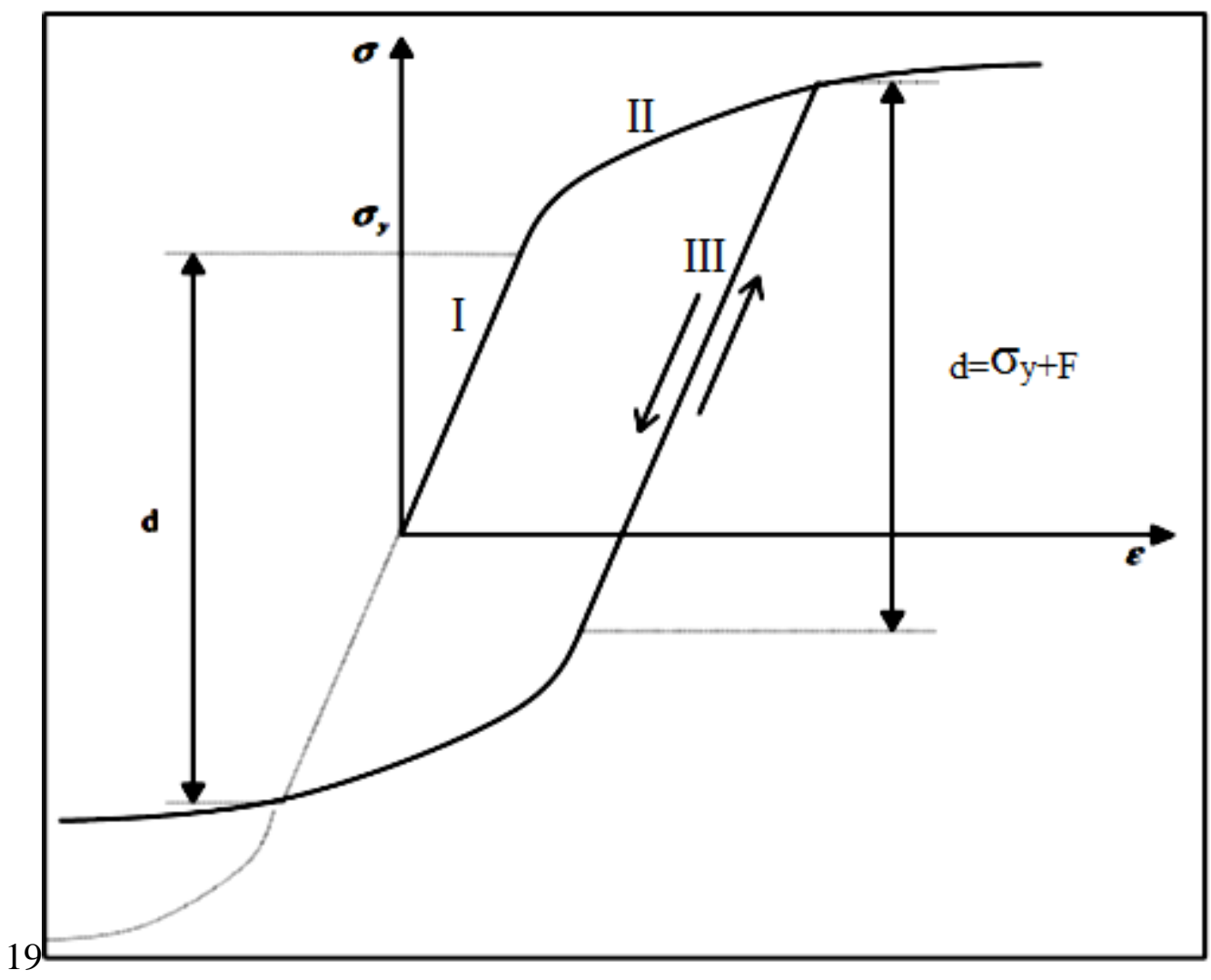

Figure 2: Non-linear kinematic hardening behavior.

The yield function in Chaboche model is as shown in Eq.1 [18]:

$F=\sqrt{\frac{3}{2}(\{s\}-\{\alpha\})^{T}[M](\{s\}-\{\alpha\})}-\sigma_{y}=0$

Where, $\{s\}$ is the deviatoric stress, $\sigma_{y}$ refers to the yield strength of the used material, $[\mathrm{M}]$ is the mass matrix, and $\{\alpha\}$ represents the back stress which can be calculated as follows:

$$
\begin{aligned}
& \{\alpha\}=\sum_{i=1}^{n}\left\{\alpha_{i}\right\} \\
& \{\Delta \alpha\}_{i}=\frac{2}{3}\left\{\Delta \varepsilon^{p}\right\}-\gamma_{i}\left\{\alpha_{i}\right\} \Delta \hat{\varepsilon}^{p}+\frac{1}{C_{i}} \frac{d C_{i}}{d T} \Delta T\{\alpha\}
\end{aligned}
$$

where, $\hat{\varepsilon}^{p}$ is accumulated plastic strain, $\mathrm{T}$ is the temperature, $\mathrm{C}_{\mathrm{i}}, \gamma_{\mathrm{i}}$ are the Chaboche material parameters for $n$ number of pairs. 
To determine $C_{1}$ and $\gamma_{1}$ constants for a known plastic strain- back stress behavior, one may consider back stress relation as:

$\alpha_{i}=\frac{C_{1}}{\gamma_{1}}\left(1-e^{-\gamma_{1} \varepsilon_{i}{ }^{p}}\right)$

Although, this expression assumes zero for initial back stress, the first data point may use the following equation for non-zero back stress.

$\alpha_{i}=\frac{C_{1}}{\gamma_{1}}\left(1-e^{-\gamma_{1} \varepsilon_{i}^{p}}\right)+\alpha_{1} e^{-\gamma_{1} \varepsilon_{i}{ }^{p}} \quad$ for $\mathrm{i}>1$

Same procedure can be used to obtain $C_{i}$ and $\gamma_{i}$ for higher levels of Chaboche Model.

Considering von-Mises yielding criteria, finite element models in the environment of ANSYS WORKBENCH 14.5 are constructed to calculate the residual hoop stress in an autofrettaged mono-block cylinder (one cycle of autofrettage on the inner surface). These models are used for a cylinder with inner and outer diameters of 146 $\mathrm{mm}, 305 \mathrm{~mm}$, is made of the NiCrMoV125 steel. The material constants are: $\mathrm{E}=268$ $\mathrm{GPa}, v=0.29, \rho=7800 \mathrm{~kg} / \mathrm{m} 3$ and $\sigma_{y}=700 \mathrm{MPa}$, where, $\rho, v$, and $\sigma_{\mathrm{y}}$ are the density, Poisson's ratio and yield stress, respectively. For the bilinear kinematic model the slope of the straight line in the plastic region has been taken as: $\mathrm{H}=75 \mathrm{GPa}$. Also, for a two level of nonlinear Chaboche model, the material parameters have been taken as: $\mathrm{C}_{1}=75 \mathrm{GPa}, \gamma_{1}=-50, \mathrm{C}_{2}=25 \mathrm{Gpa}, \gamma_{2}=30$ - see Figure 3 .

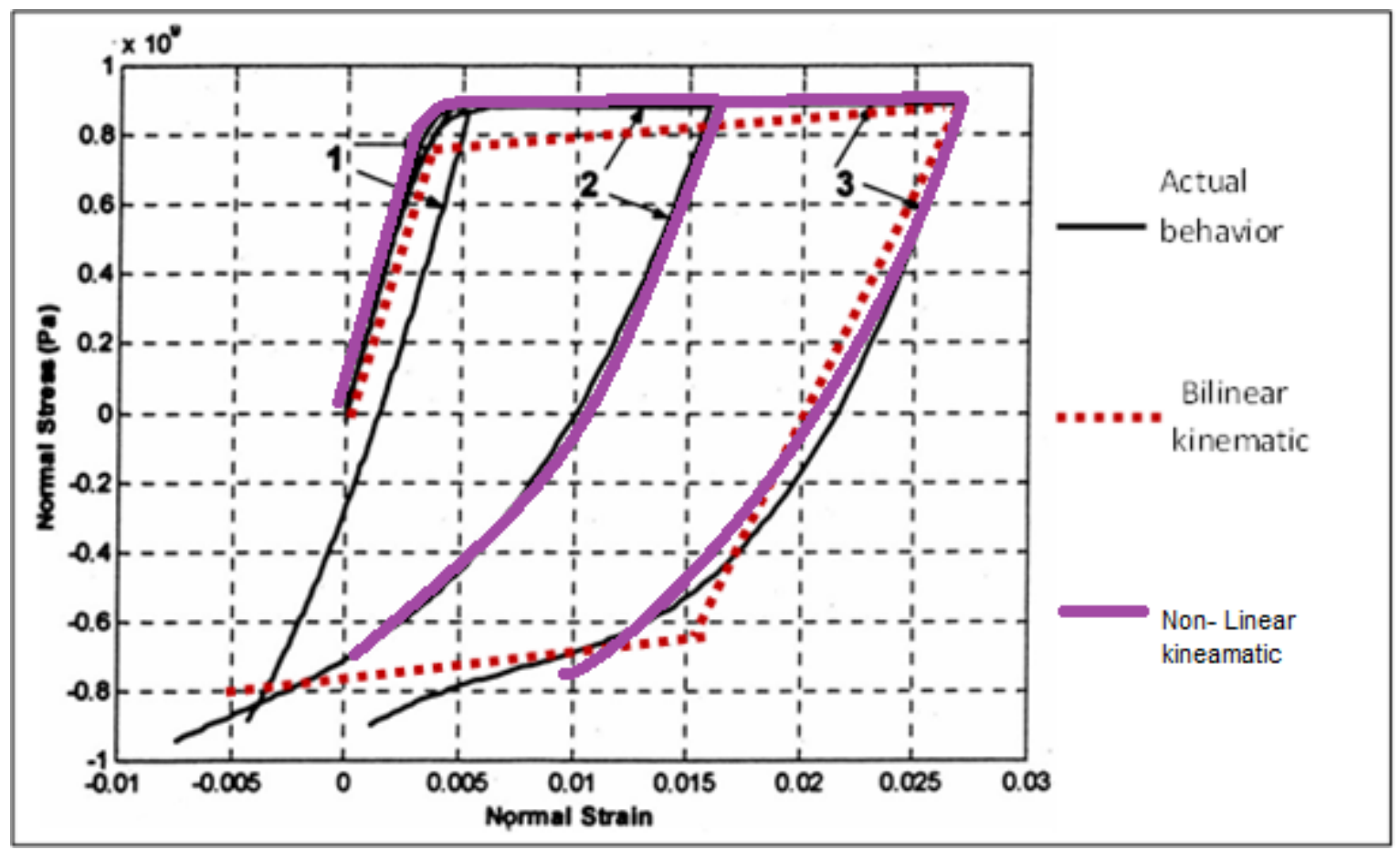

Figure 3: Actual, bilinear, and non-linear kinematic stress-strain behavior. 
To verify these finite element models, the residual hoop stress distribution through the wall thickness is calculated for each model and then compared with that based on actual material behavior presented in work by Jahed and Ghanbari [10], as shown in Figure 4.

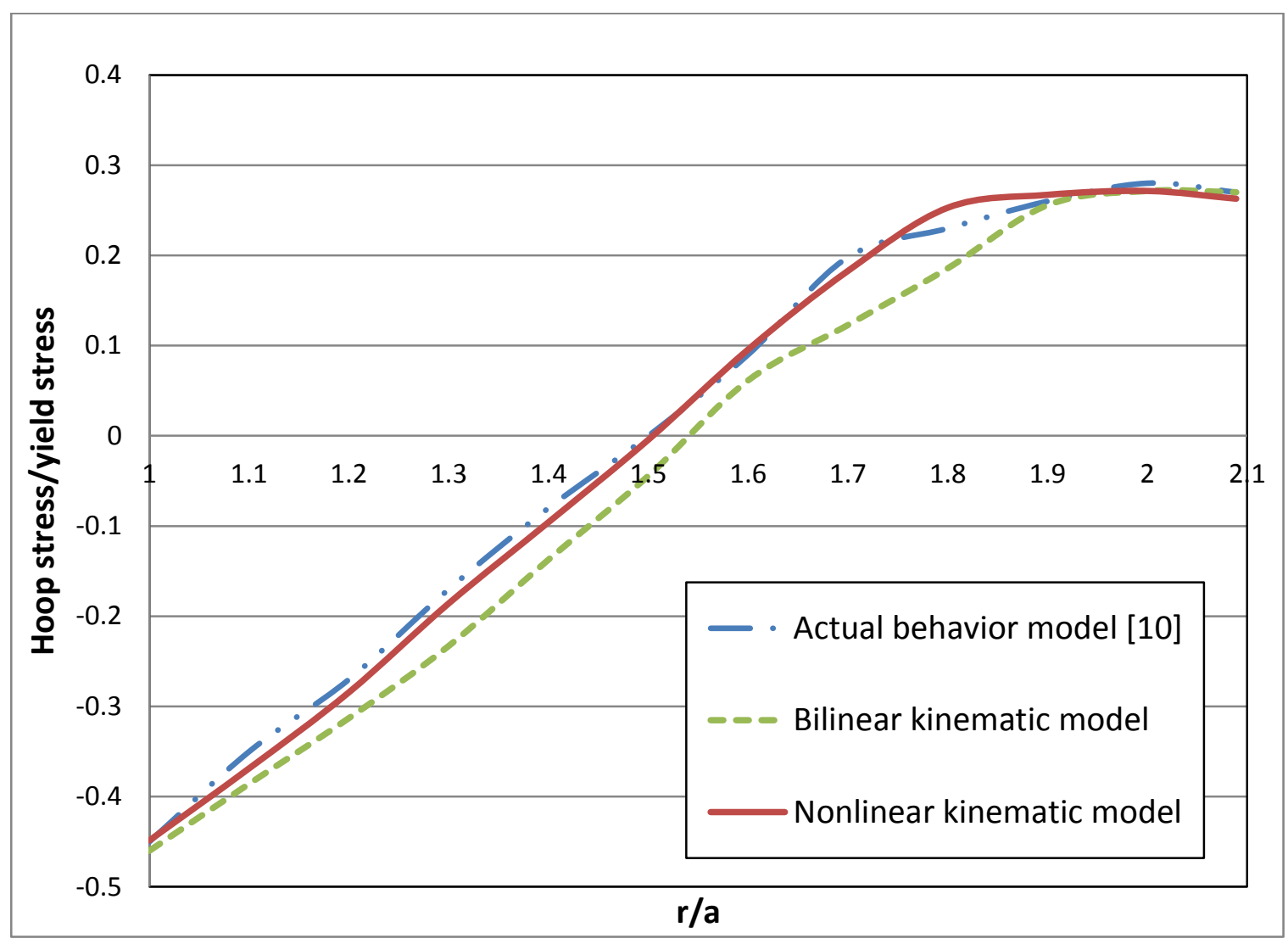

Figure 4: Residual hoop stresses for different models.

In Figure $4, r$ is the radial position and $a$ is the inner radius. The results shown in Figure 4 clearly demonstrate that the predicted residual hoop stresses based on either the bilinear kinematic hardening or nonlinear kinematic hardening models have quite good agreement with the results based on the actual behavior, However the second model is much agreeable than the first one. On the base of these findings and the fact that the nonlinear kinematic model is usually used for cyclic plasticity, the nonlinear (Chaboche) kinematic hardening model has to be utilized for multiple autofrettage.

\section{NEW COMBINATIONS OF MULTIPLE AUTOFRETTAGE}

Using the proposed finite element nonlinear kinematic hardening model, new combinations of autofrettage process on the outer and inner surface have been developed to improve the radial distribution of residual stresses of the autofrettaged cylinders by increasing the beneficial compressive residual stresses at the inner part of the cylinder and decreasing the harmful tensile residual stresses at the outer part of the cylinder. These new combinations can be summarized as: 
Combination 1: One autofrettage cycle subjected to the outer surface of the cylinder followed by three cycles of autofrettage subjected to the inner surface and the autofrettage pressure for these three cycles has the same value.

Combination 2: The same as combination one, but the three inner autofrettage pressures have ascending values.

Combination 3: The same as combinations one and two, but the three inner autofrettage pressures have descending values.

Combination 4: Three cycles of double (external prior to internal) autofrettage processes have the same autofrettage pressure values on either the outer surface or the inner surface of the cylinder for each cycle.

Combination 5: The same as combination four, but the three double autofrettage cycles have ascending values of autofrettage pressure.

Combination 6: The same as combinations four and five, but the three double autofrettage cycles have descending values of autofrettage pressure.

\section{RESULTS AND DISCUSSION}

For the same cylinder used in section 2, the residual hoop stresses have been evaluated for the prementioned combinations. For the sake of expose the improvement, the residual stresses after each cycle for each combination have been illustrated and then compared with the calculated residual stress due to the traditional inner surface single cycle autofrettage, as shown in Figures 5 through 10. The values of autofrettage pressures for each combination are as shown in Table 1.

Table 1: Values of autofrettage pressures for each combination

\begin{tabular}{|c|c|c|c|c|}
\hline Cycles & Outer surface & Cycle 1, & Cycle 2, & Cycle 3, \\
\hline Combination & pressure, MPa & $\mathrm{MPa}$ & $\mathrm{MPa}$ & $\mathrm{MPa}$ \\
\hline 1 & 730 & 500 & 500 & 500 \\
\hline 2 & 730 & 300 & 400 & 500 \\
\hline 3 & 730 & 500 & 400 & 300 \\
\hline 4 & - & $\begin{array}{l}\text { (Double) } \\
730,500\end{array}$ & $\begin{array}{l}\text { (Double) } \\
730,500\end{array}$ & $\begin{array}{l}\text { (Double) } \\
730,500\end{array}$ \\
\hline 5 & - & $\begin{array}{l}\text { (Double) } \\
530,300\end{array}$ & $\begin{array}{l}\text { (Double) } \\
630,400\end{array}$ & $\begin{array}{l}\text { (Double) } \\
730,500\end{array}$ \\
\hline 6 & - & $\begin{array}{l}\text { (Double) } \\
730,500\end{array}$ & $\begin{array}{l}\text { (Double) } \\
630,400\end{array}$ & $\begin{array}{l}\text { (Double) } \\
530,300\end{array}$ \\
\hline
\end{tabular}




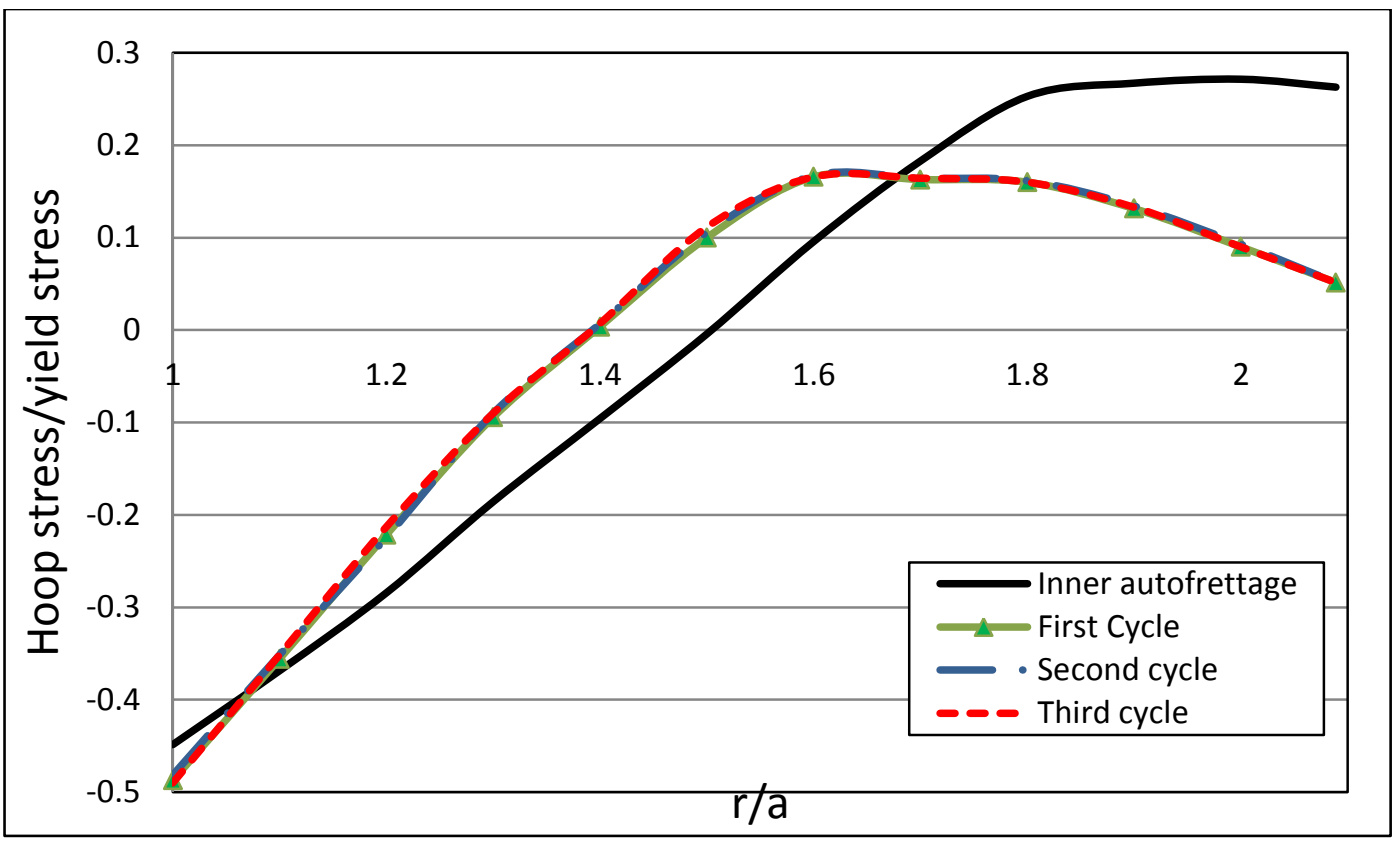

Figure 5: Radial distribution for residual hoop stresses for Combination 1.

It is apparent from Figure 5 that combination 1 can improve the radial distribution of residual hoop stress by increasing the compressive stresses at the near bore area and decreasing the tensile residual stresses at the outer part of the cylinder. It should be noted that while the autofrettage cycles on the inner surface have the same autofrettage pressure there is no benefit of the second and third cycles over the first one. That may be compatible with the conclusion of Jahed et al. [2]. That may be compatible with the conclusion of Jahed et al. [2].

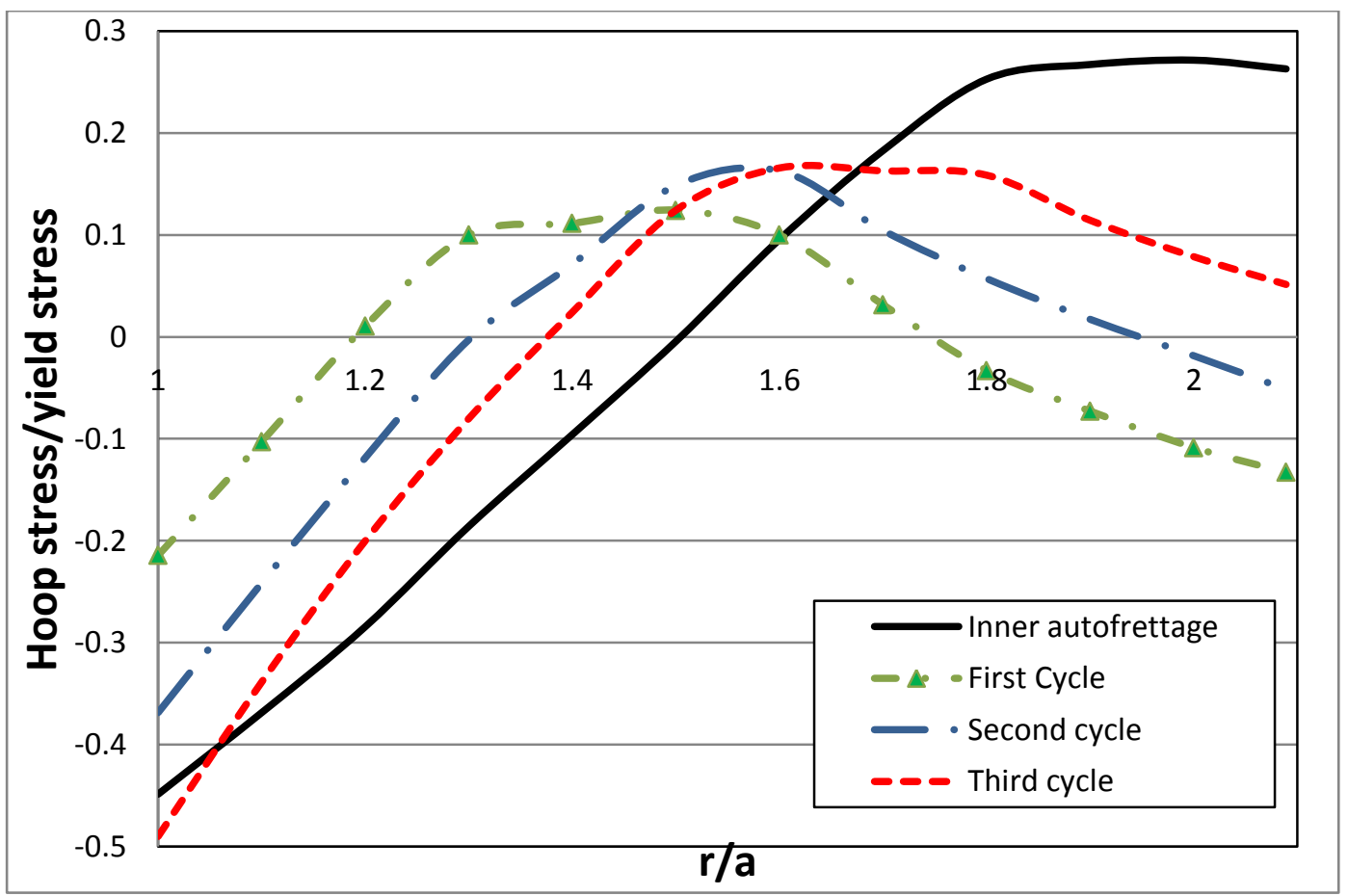

Figure 6: Radial distribution for residual hoop stresses for Combination 2. 
Figure 6 shows that the autofrettage cycles on the inner surface with ascending autofrettage pressure can improve the residual hoop stress by increasing the number of these cycles. However the increase of the beneficial compressive stress is not remarkable, the decrease in tensile stress is apparent compared with the residual stresses due to the traditional single inner cycle of autofrettage.

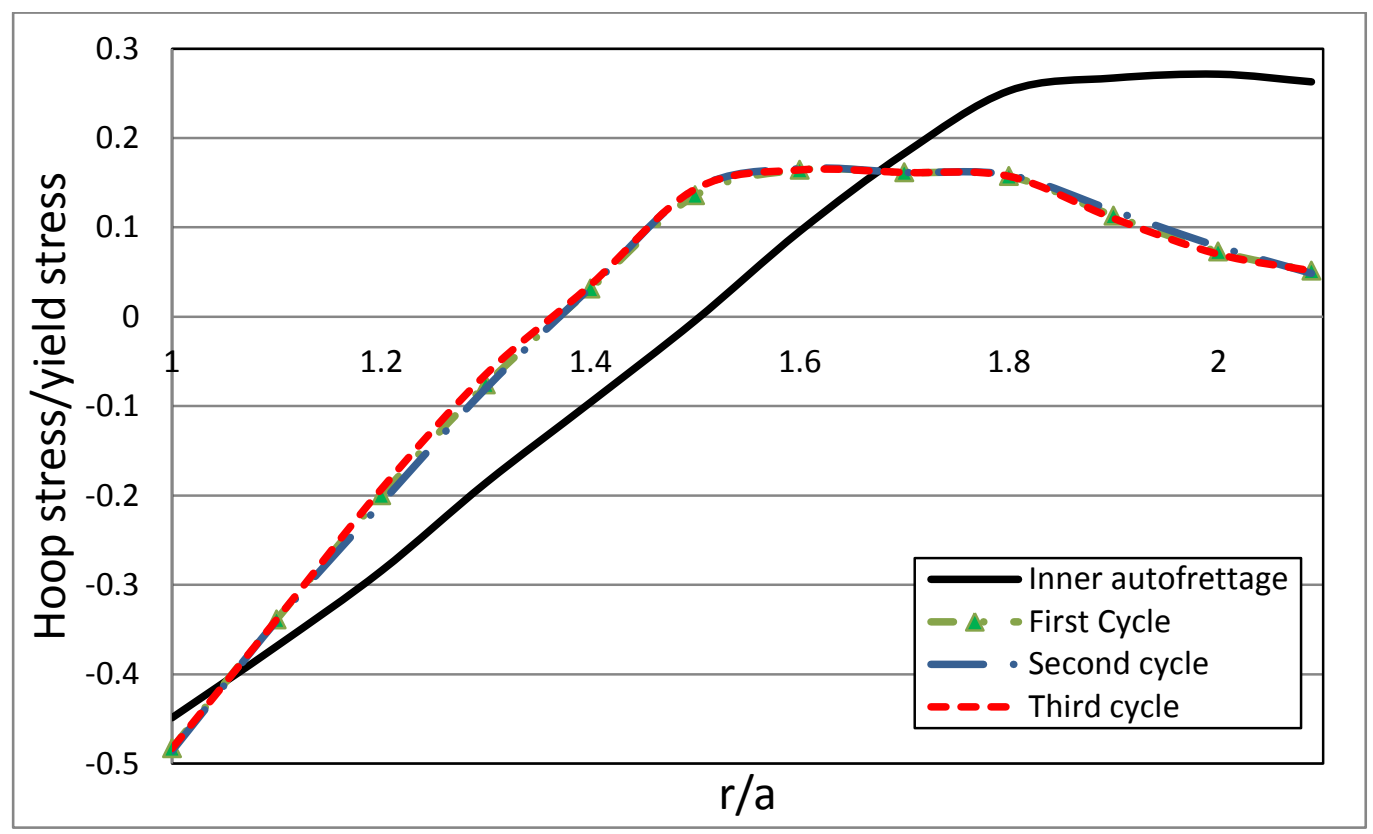

Figure 7: Radial distribution for residual hoop stresses for Combination 3.

From Figure 7, one can observe that there is no improvement in the residual stresses with increasing of the number of cycles which have descending autofrettage pressures.

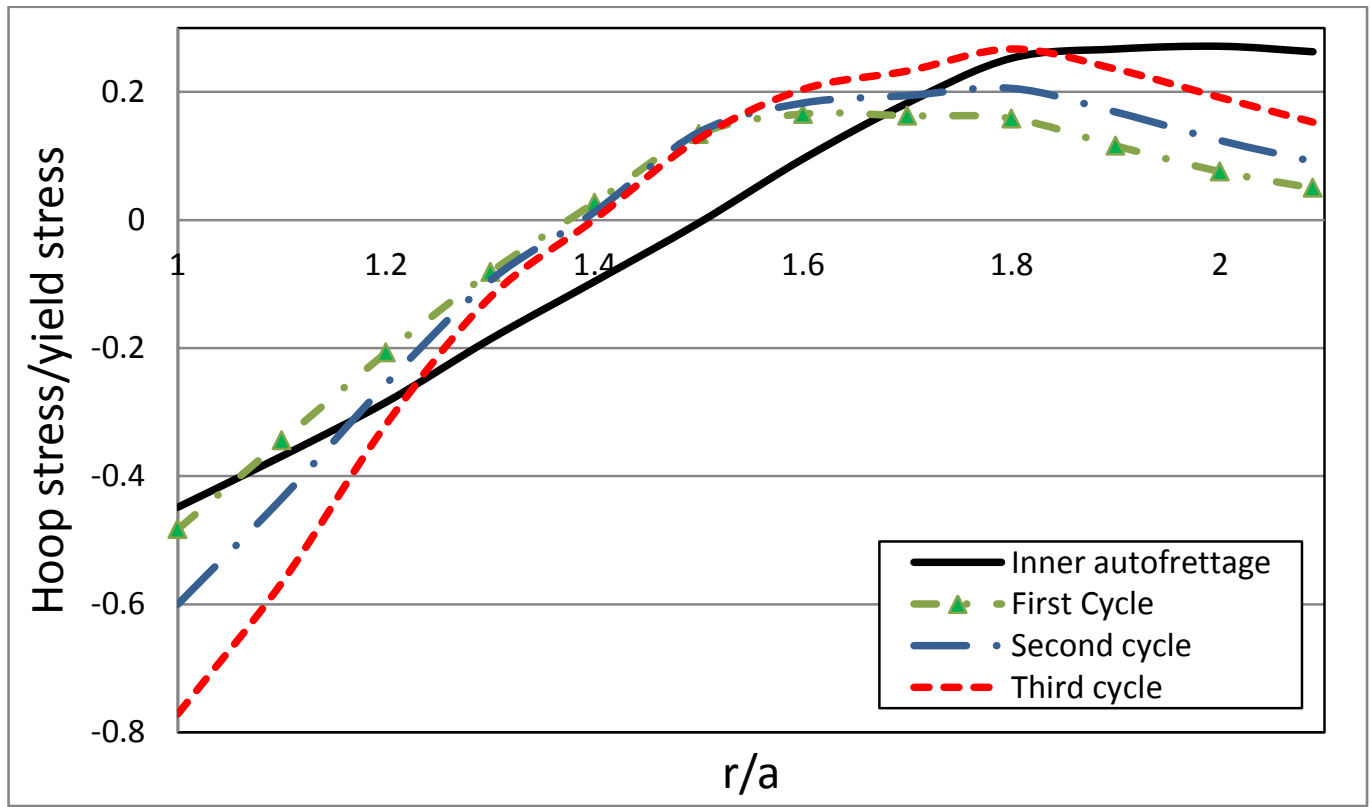

Figure 8: Radial distribution for residual hoop stresses for Combination 4. 
Examination of Figure 8 reveals that cyclic double autofrettage processes have an obvious effect on improvement of the radial distribution of residual stresses. This combination increases the compressive residual stresses considerably, especially at the inner bore area (working area), and slightly increase the harmful tensile residual stresses at the outer part of the cylinder.

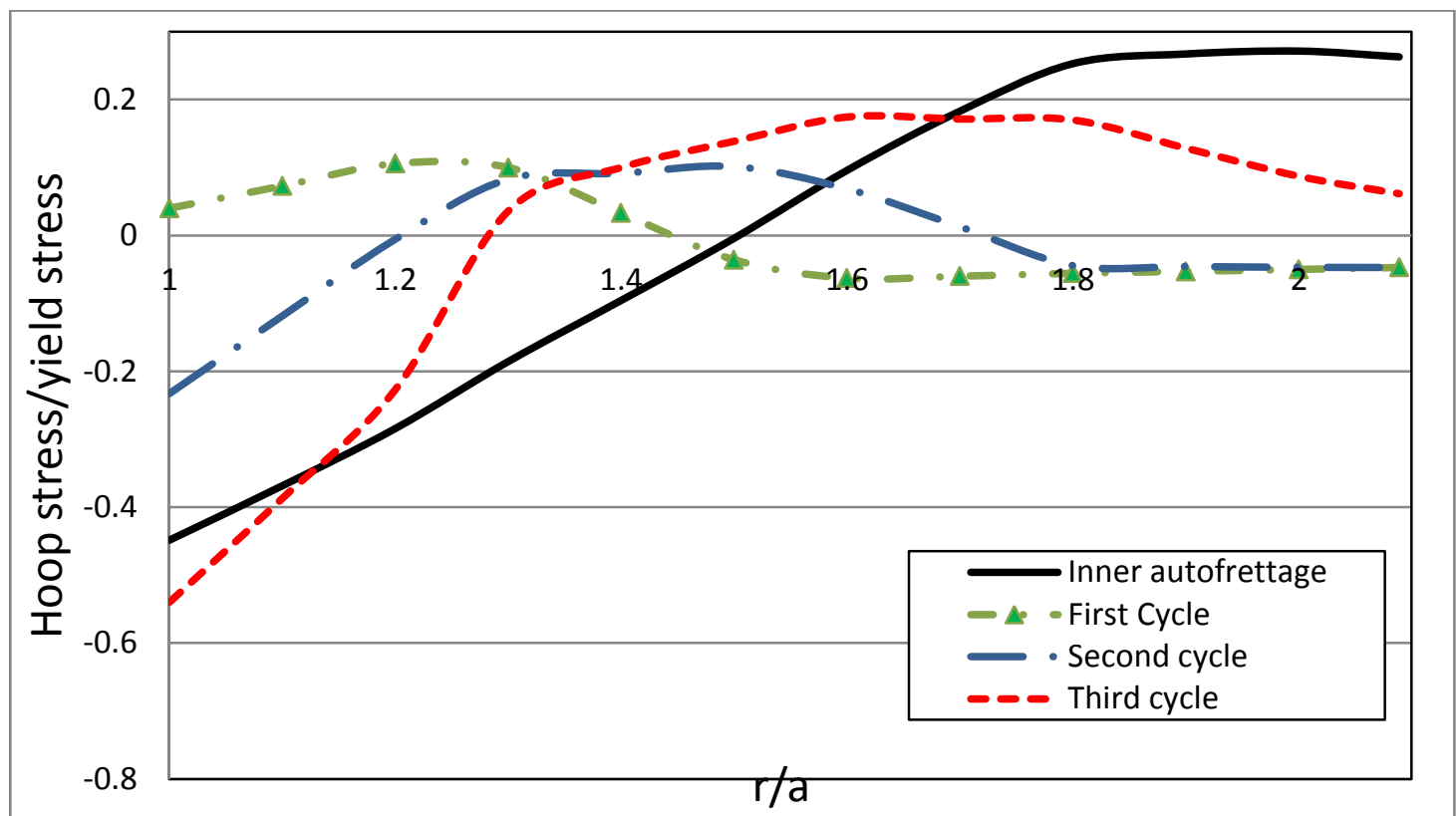

Figure 9: Radial distribution for residual hoop stresses for Combination 5.

It has been observed from Figure 9 that the effect of double autofrettage cycles with ascending autofrettage pressure has slightly consequence in improving the residual stresses.

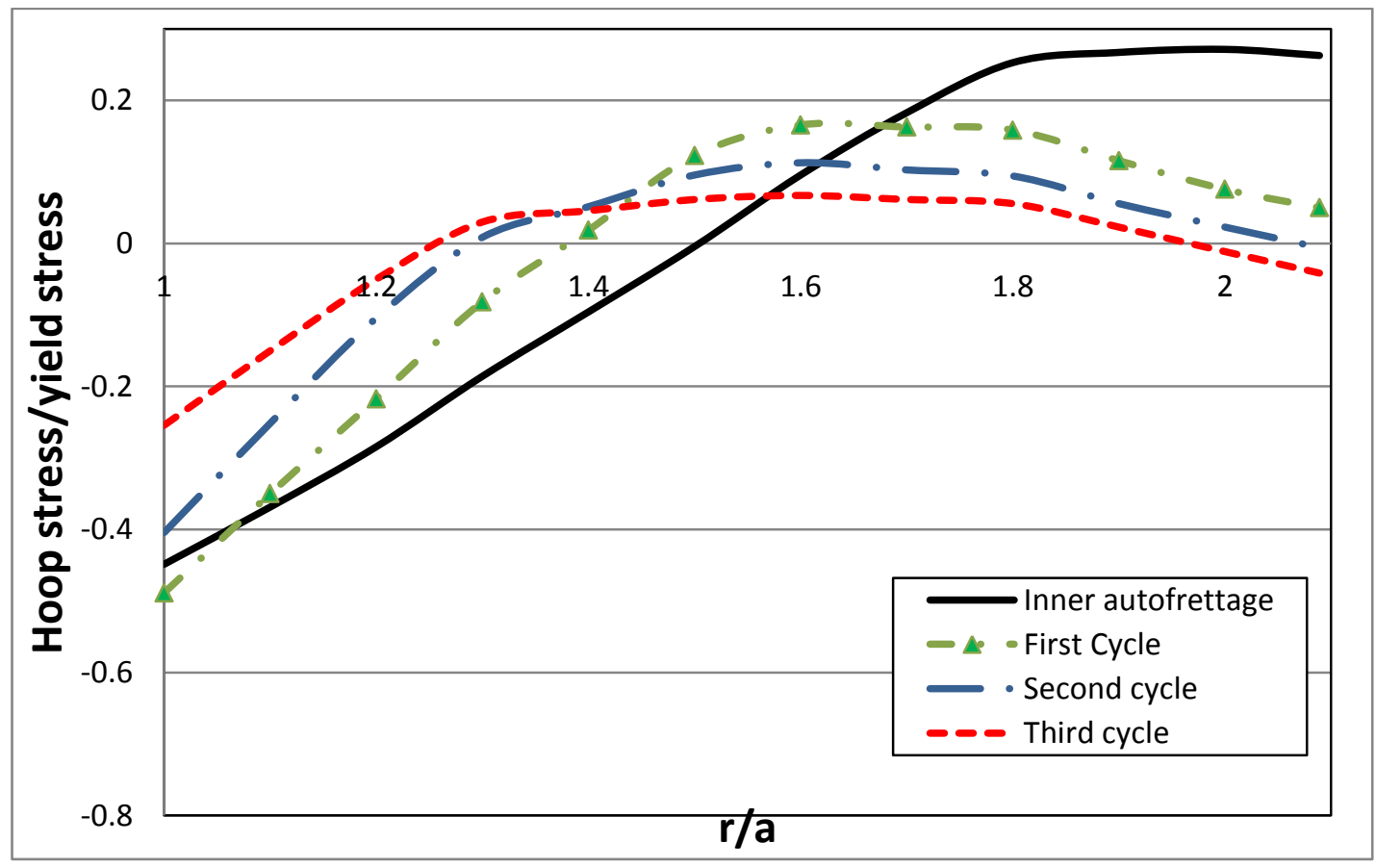

Figure 10: Radial distribution for residual hoop stresses of Combination 6. 
Figure 10 shows that the double autofrettage cycles with descending autofrettage pressures cause losing of the benefit of double autofrettage and impair the distribution of the residual stresses.

Usually any autofretted cylinder can withstand internal working pressure around the value of autofrettage pressure. Therefore the equivalent Von-Mises stress for all prementioned combinations (after the third cycle) have been calculated under the effect of $500 \mathrm{MPa}$ working pressure to demonstrate which combinations behave elastically under this high operational load. Figure 11 provides Von-Mises stress distribution along the wall thickness for all different combinations compared with stresses for a cylinder subjected to inner single autofrettage cycle and with stresses for a mono-block virgin cylinder which has no residual stresses.

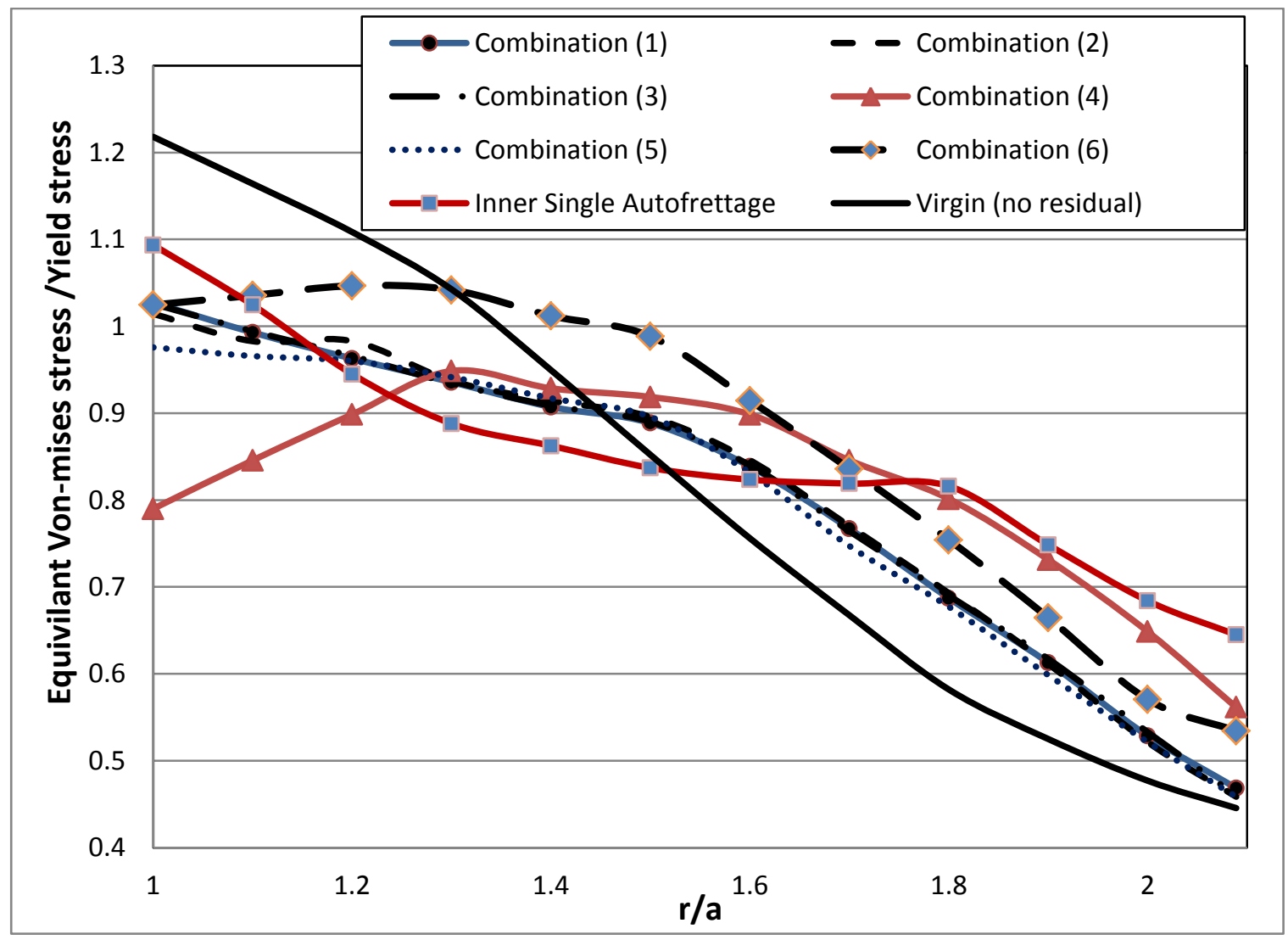

Figure 11: Radial distribution for Von-Mises stresses subjected to 500Mpa working pressure for different combinations.

It is clear from Figure 11 that combinations 4 and 5 are the only cylinders which behave elastically when subjected to $500 \mathrm{MPa}$ working pressure. It is also obvious that combination four can withstand more operational load.

\section{SUMMARY AND CONCLUSIONS}

This paper addresses new techniques of cyclic autofrettage and double autofrettage processes. The residual stresses for these new combinations have been evaluated using nonlinear kinematic hardening finite element model and then compared with 
those due to the conventional inner single cycle autofrettage for a thick wall cylindrical pressure vessel.

It has been proved that the nonlinear kinematic hardening model is more accurate than the bilinear kinematic hardening model if the residual stress distributions based on both models are compared with those based on the actual stress strain behavior of the material. Moreover the nonlinear kinematic hardening model gives the ability to consider the effect of cyclic plasticity. Also, it is found that most of these new combinations could enhance the residual hoop stress over the whole thickness of the cylinder wall.

The conclusion's highlights for these new combinations are summarized below as:

1- For combinations one and three, there is no need for cyclic autofrettage processes, as the residual stresses do not improve with sequenced autofrettage cycles.

2- In contrast, combinations two, four and five provide enhancement of the residual stress with increasing of number of autofrettage or double autofrettage cycles.

3- In a different way, Combination 6 losses the residual stresses specially at the near bore area with the increase of double autofrettage cycles.

4- Combination 4 is the most recommended as it provides high improvement in the compressive residual stresses at the inner part of the cylinder with the increase of the double autofrettage cycles, besides it affords slightly increase in the nondesirable tensile stress at the outer part of the cylinder with increase of number of cycles.

Regarding cyclic autofrettage and double autofrettage processes, one can conclude that these techniques are very promising to be used in improving the radial distribution of the residual hoop stresses.

\section{REFERENCES}

[1] Parker, A. P., 2004, "A Re-Autofrettage Procedure for Mitigation of Bauschinger Effect in Thick Cylinders," ASME, Journal of Pressure Vessel Technology, 126, pp. 451-454.

[2] Jahed, H. Ahmadi, B. and Shambooli, M., 2006, "Re-Autofrettage", ASME, Journal of Pressure Vessel Technology, 128, pp. 223-226.

[3] Parker, A. P., 2001, "Bauschinger Effect Design Procedures for Compound Tubes Containing an Autofrettaged Layer," ASME, Journal of Pressure Vessel Technology, 123, pp. 203-206.

[4] Parker, A. P. and Kendall, D. P., 2003, "Residual Stresses and Lifetimes of Tubes Subjected to Shrink Fit Prior to Autofrettage," ASME, Journal of Pressure Vessel Technology, 125, pp. 282-286.

[5] Majzoobi, G. H. Farrah, G.H. Pipelzadeh, M. K. and Akbari, A., 2004, "Experimental and Finite Element Prediction of Bursting Pressure in Compound Cylinder," International Journal of Pressure Vessels and Piping, 81, pp.889896,.

[6] Jahed, H. Farshi, B. and Karimi, M., 2006, " Optimum Autofrettage and ShrinkFit Combination in Multi-Layer Cylinders," ASME, Journal of Pressure Vessel Technology, 128, pp. 196-200. 
[7] Parker, A. P., 2012, "Compound and Monoblock Cylinders Incorporating Reverse-Autofrettage to Reduce External Hoop Stresses," Paper PVP201278298, Proceedings of ASME PVP2012 Pressure Vessels and Piping Division Conference July 15-19, Toronto, Ontario, Canada.

[8] Ossama Abdelsalam and Ramin Sedaghti, 2013, "Design Optimization of Compound Cylinders Subjected to Autofrettage and Shrink-Fitting Processes," ASME, Journal of Pressure Vessel Technology, 135(2), pp. 021209 1-8.

[9] Ossama Abdelsalam and Ramin Sedaghti, 2013, "Outer surface prior to inner surface Double Autofrettage technique for compound cylinders," ASME, pressure vessel and piping conference, Paris, France, pp. 97035 1-6.

[10] Jahed, H., and Ghanbari, G., "Actual Unloading Behavior and Its Significance on Residual Stress in Machined Autofrettaged Tube", ASME J. Pressure Vessel Technol., vol. 125, pp. 321-235, 2003.

[11] Huang, X P., 2005, "A General Autofrettage Model of A Thick-Walled Cylinder Based on Tensile-Compressive Stress-Strain Curve of a Material", Journal of Strain Analysis for Engineering Design, vol. 40, No. 6, pp.599-607.

[12] Huang, X. and Moan, T., 2009, " Residual Stress in an Autofrettaged Tube Taking Bauschinger Effect as a Function of the Prior Plastic Strain", ASME ,Journal of Pressure Vessel Technology, Vol. 131, pp. 021207-1-7.

[13] Md. Tanjin Amin, Abu Rayhan Md. Ali, Tousif Ahmed and Faisal Ahmed, 2013, "Optimum Design of Autofrettaged Thick-Walled Cylinders," Global Journal of Researches in Engineering Mechanical and Mechanics Engineering (A), Vol. 13 Issue 8 Version 1.0.

[14] G.H. Farrahi , George Z. Voyiadjis, S.H. Hoseini, E. Hosseinian, 2012, "Residual Stress Analyses of Re-Autofrettaged Thick-Walled Tubes," International Journal of Pressure Vessels and Piping Vol. 98, pp.57-64.

[15] Chaboche, J. L., Dang-Van, K. and Cordier, G., 1979, "Modelization of the Strain Memory Effect on the Cyclic Hardening of 316 Stainless Steel,"” SMIRT5, Division L Berlin.

[16] Chaboche, J. L., 1989, "Constitutive Equations for Cyclic Plasticity and Cyclic Viscoplasticity," International Journal of Plasticity, Vol. 5, pp. 247-251.

[17] Chaboche, J. L., 1991, "On Some Modifications of Kinematic Hardening to Improve the Description of Ratchetting Effects,"” International Journal of Plasticity, Vol. 7، pp.661- 669.

[18] Abdullah Aziz Saad, 2012, Cyclic plasticity and creep of power plant material. $\mathrm{PhD}$. Thesis, University of Nottingham, UK. 